\title{
On solutions of polynomial congruences
}

\author{
by
}

\section{SANOLI Gun (Chennai)}

1. Introduction. An interesting problem in number theory is to find solutions of polynomial congruences. In a recent work [9], Ram Murty considered the polynomial congruence $x^{q} \equiv a(\bmod p)$, where $p$ is a prime, $q$ is a divisor of $p-1$ and $a^{(p-1) / q} \equiv 1(\bmod p)$. He showed that the smallest solution $x_{0}$ of the congruence is $\ll p^{3 / 2}(\log p) / q$. In this paper, we consider consecutive solutions of that congruence when $a=1$. We show that for a natural number $\mathrm{M}$, the above polynomial congruence has $\mathrm{M}$ consecutive solutions for sufficiently large primes $p$. More precisely, we prove

THEOREM 1.1. Let $p$ be an odd prime and $\mathrm{M}$ be a natural number such that $p>2^{4 \mathrm{M}} \mathrm{M}^{4}$. Further, let $q$ be a prime divisor of $p-1$ with $q>$ $(p-1)^{1-1 / 4 \mathrm{M}}$. Then the congruence

$$
x^{q} \equiv 1(\bmod p)
$$

has $\mathrm{M}$ consecutive solutions.

We also consider two-fold generalizations of the question investigated by Ram Murty. In one direction, we study polynomial congruences of the type

$$
x^{q} \equiv a(\bmod d),
$$

where $d$ is not necessarily prime, and in another direction, we consider congruences of the form

$$
f(x)^{q} \equiv a(\bmod p), \quad(a, p)=1,
$$

where $f(x) \in(\mathbb{Z} / p \mathbb{Z})[x]$. In particular, we prove the following theorems:

Theorem 1.2. Let $q$, d be natural numbers such that $q \mid \phi(d)$. Also let $n(q)$ be the number of elements in $(\mathbb{Z} / d \mathbb{Z})^{*}$ whose order divides $q$. Suppose that the polynomial congruence

$$
x^{q} \equiv a(\bmod d)
$$

2010 Mathematics Subject Classification: 11T06, 11L40, 11T24.

Key words and phrases: polynomial congruences, character sums. 
has a solution. Then the smallest solution $x_{0}$ satisfies

$$
\left|x_{0}\right| \ll \frac{d^{1 / 2} \phi(d) \log d}{n(q)} .
$$

Note that Theorem 1.2 is non-trivial for $n(q) \gg d^{1 / 2+\varepsilon}$. As an immediate consequence, we have

Corollary 1.3. Let $p$ be an odd prime, $d=p^{n}, 2 p^{n}$ and $q \mid \phi(d)$. Suppose that the polynomial congruence

$$
x^{q} \equiv a(\bmod d)
$$

has a solution. Then the smallest solution $x_{0}$ satisfies

$$
\left|x_{0}\right| \ll \frac{p^{3 n / 2} n \log p}{q} .
$$

REMARK 1.1. The case $n=1$ in the above corollary is a theorem of Ram Murty (see [9]).

TheOREM 1.4. Let $p, q$ be primes such that $q \|(p-1)$. Also let $f(x)$ be a polynomial over $\mathbb{Z} / p \mathbb{Z}$ which has $m$ distinct roots and $(\ell, \operatorname{deg} f)=1$ for any $\ell \mid(p-1) / q$. Suppose that the polynomial congruence

$$
f(x)^{q} \equiv a(\bmod p), \quad(a, p)=1,
$$

has a solution. Then the smallest solution $x_{0}$ satisfies

$$
\left|x_{0}\right| \ll \frac{m p^{3 / 2} \log p}{q} .
$$

REMARK 1.2. Putting $f(x)=x$ in Theorem 1.4, we again recover the theorem of Ram Murty (see [9]). We also refer to a related article due to Hudson [6].

Next we study the distribution of the roots (if they exist) of the congruence $x^{q} \equiv a(\bmod d)$ with $(a, d)=1$. We list the $n(q)$ roots as $r_{1}<\cdots<$ $r_{n(q)}<d$. In this context, we have the following theorem:

TheOREM 1.5. Fix $\alpha \in(0,1), \delta>0$ and a natural number $d$. Suppose that $q \mid \phi(d)$ and $n(q)>d^{\delta}$. Then there exists an $\varepsilon(\delta)>0$ such that $\#\left\{r_{i} \mid r_{i}^{q} \equiv a(\bmod d), 0<r_{i}<\alpha d, 1 \leq i \leq n(q)\right\}=n(q) \alpha+O\left(n(q) d^{-\varepsilon(\delta)}\right)$. In particular, if there is a solution of $x^{q} \equiv a(\bmod d)$, then the smallest solution $x_{0}$ is $\ll d^{1-\varepsilon(\delta)}$.

As an immediate corollary, we have

Corollary 1.6. Fix $\alpha \in(0,1), \delta>0$ and $d=p^{n}, 2 p^{n}$ with $p$ odd prime. Suppose that $q \mid \phi(d)$ and $q>d^{\delta}$. Then there is $\varepsilon(\delta)>0$ such that

$$
\#\left\{r_{i} \mid r_{i}^{q} \equiv a(\bmod d), 0<r_{i}<\alpha d, 1 \leq i \leq q\right\}=q \alpha+O\left(q d^{-\varepsilon(\delta)}\right) .
$$


2. Preliminaries. Throughout the paper $p$ is prime, $\mathrm{M}, \mathrm{V}, \ell, q, d$ are natural numbers, $\chi_{0}$ is the principal character modulo $p$ or $d$ depending on the context. First we shall need the following estimate due to Weil [11].

Theorem 2.1 (Weil). For an integer $\ell$ satisfying $2 \leq \ell<p$ and for any non-principal characters $\chi_{1}, \ldots, \chi_{\ell}$ and distinct $a_{1}, \ldots, a_{\ell} \in \mathbb{Z} / p \mathbb{Z}$, we have

$$
\left|\sum_{n=1}^{p} \chi_{1}\left(n+a_{1}\right) \cdots \chi_{\ell}\left(n+a_{\ell}\right)\right| \leq(\ell-1) \sqrt{p} .
$$

For $\ell=2$, Davenport [4] proved the above bound. Note that when $\ell=1$, the above sum is 0 . Using this, we prove the following lemma. of

LEMma 2.2. Let $N(p, \mathrm{M})$ denote the number of $\mathrm{M}$ consecutive solutions

$$
x^{q} \equiv 1(\bmod p)
$$

Then

$$
\left|N(p, \mathrm{M})-p\left(\frac{q}{p-1}\right)^{\mathrm{M}}\right| \leq 2^{\mathrm{M}} \mathrm{M} \sqrt{p} .
$$

Proof. Write

$$
N(p, \mathrm{M})=\sum_{n=1}^{p} \prod_{j=0}^{\mathrm{M}-1}\left(\frac{1}{p-1} \sum_{\chi} \bar{\chi}(1) \chi\left((n+j)^{q}\right)\right),
$$

where the inner sum is over all characters modulo $p$. Dividing the sum into two parts, with $\chi^{q}=\chi_{0}$ and $\chi^{q} \neq \chi_{0}$, we have

$$
N(p, \mathrm{M})=(p-1)^{-\mathrm{M}} \sum_{n=1}^{p} \prod_{j=0}^{\mathrm{M}-1}\left(q+\sum_{\substack{\chi \\ \chi^{q} \neq \chi_{0}}} \chi\left((n+j)^{q}\right)\right)=p\left(\frac{q}{p-1}\right)^{\mathrm{M}}+A,
$$

where

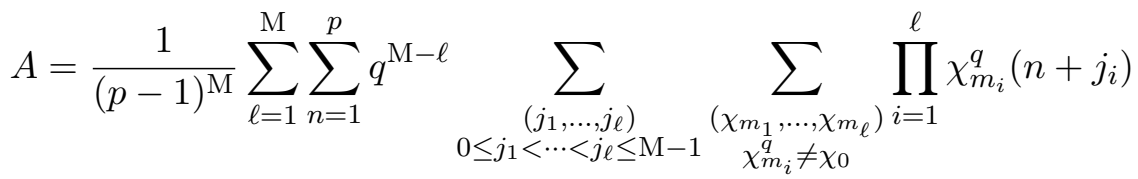

$$
\begin{aligned}
& =\sum_{\ell=1}^{\mathrm{M}}\left(\frac{q}{p-1}\right)^{\mathrm{M}-\ell} \sum_{\substack{\left(j_{1}, \ldots, j_{\ell}\right) \\
0 \leq j_{1}<\cdots<j_{\ell} \leq \mathrm{M}-1}} \frac{1}{(p-1)^{\ell}} \sum_{\substack{\left.\chi_{m_{1}}, \ldots, \chi_{m_{\ell}}\right) \\
\chi_{m_{i}} \neq \chi_{0}}} \sum_{n=1}^{p} \prod_{i=1}^{\ell} \chi_{m_{i}}^{q}\left(n+j_{i}\right) .
\end{aligned}
$$

Hence by using the estimate of Weil (Theorem 2.1), one has

$$
|A| \leq \mathrm{M} \sqrt{p} \sum_{\ell=1}^{\mathrm{M}}\left(\begin{array}{c}
\mathrm{M} \\
\ell
\end{array}\right)\left(\frac{q}{p-1}\right)^{\mathrm{M}-\ell} \leq 2^{\mathrm{M}} \mathrm{M} \sqrt{p}
$$


We refer to [5] where the estimate of Weil has been exploited in another context. We shall need the following generalization of the Pólya-Vinogradov theorem for proving Theorems 1.2 and 1.4 .

LEMMA 2.3. If $\chi\left(\neq \chi_{0}\right)$ is an $\ell$ th order character to the prime modulus $p$ and if $f(x)$ is a polynomial over $\mathbb{Z} / p \mathbb{Z}$ which has $m$ distinct roots and $(\ell, \operatorname{deg} f)=1$, then

$$
\sum_{n \leq T} \chi(f(n)) \ll m \sqrt{p} \log p \quad \text { for } 1 \leq T \leq p .
$$

To prove Lemma 2.3 , we need the following consequence of the works of Weil [12, 13] (see also [2] and page 45 of [10]).

THEOREM 2.4. Let $p$ be prime and $\chi\left(\neq \chi_{0}\right)$ be a multiplicative character of order $\ell$ with $\ell \mid(p-1)$. Suppose that $f(x)$ is a polynomial over $\mathbb{Z} / p \mathbb{Z}$ which has $m$ distinct roots and $(\ell, \operatorname{deg} f)=1$. Then

$$
\left|\sum_{n=1}^{p} \chi(f(n)) e(a n / p)\right| \leq m \sqrt{p},
$$

where $e(x)=e^{2 \pi i x}$.

Proof of Lemma 2.3. Write

$$
S(f, a)=\sum_{n=1}^{p} \chi(f(n)) e(a n / p) .
$$

Now

$$
\sum_{n \leq T} \chi(f(n))=\sum_{n=1}^{p} \chi(f(n)) \sum_{b \leq T}\left(\frac{1}{p} \sum_{a=1}^{p} e(a(n-b) / p)\right)
$$

since

$$
\frac{1}{p} \sum_{a=1}^{p} e(a m / p)= \begin{cases}1 & \text { if } m \equiv 0(\bmod p) \\ 0 & \text { otherwise }\end{cases}
$$

By interchanging the summations in (5), we have

$$
\begin{aligned}
\sum_{n \leq T} \chi(f(n)) & =\frac{1}{p} \sum_{a=1}^{p} \sum_{n=1}^{p} \chi(f(n)) e(a n / p) \sum_{b \leq T} e(-a b / p) \\
& =\frac{1}{p} \sum_{a=1}^{p} S(f, a) \sum_{b \leq T} e(-a b / p) \ll m \sqrt{p} \sum_{a=1}^{p} \frac{1}{a},
\end{aligned}
$$

by using Theorem 2.4 and the fact that

$$
\left|\sum_{b \leq T} e(-a b / p)\right| \leq \frac{1}{|\sin (\pi a / p)|} \ll \frac{p}{a} .
$$


Hence

$$
\sum_{n \leq T} \chi(f(n)) \ll m \sqrt{p} \log p .
$$

To prove Theorem 1.5, we need the following Erdős-Turán inequality (see page 8 of [8]) and a theorem of Bourgain [1].

Lemma 2.5. Let $\left\{x_{n}\right\}$ be a sequence of real numbers in $(0,1)$. For $\alpha \in$ $(0,1)$ and $\mathrm{V} \in \mathbb{N}$, let $N(\mathrm{~V}, \alpha)=\#\left\{n \leq \mathrm{V} \mid 0 \leq x_{n}<\alpha\right\}$. Then, for any natural numbers $\mathrm{M}$, one has

$$
|N(\mathrm{~V}, \alpha)-\mathrm{V} \alpha| \leq \frac{\mathrm{V}}{\mathrm{M}+1}+3 \sum_{m=1}^{\mathrm{M}} \frac{1}{m}\left|\sum_{n \leq \mathrm{V}} e\left(m x_{n}\right)\right| .
$$

REMARK 2.1. The constant 3 in the above estimate has been improved to 1 by Mauduit, Rivat and Sárközy [7]. The original inequality without explicit constants is due to Davenport [4].

Theorem 2.6 (Bourgain). Fix $\delta>0$ and a natural number $d$. For any subgroup $\mathrm{H}$ of $(\mathbb{Z} / d \mathbb{Z})^{*}$ with order $>d^{\delta}$, there is an $\varepsilon^{\prime}(\delta)>0$ such that

$$
\left|\sum_{x \in \mathrm{H}} e(a x / d)\right| \ll|\mathrm{H}| d^{-\varepsilon^{\prime}(\delta)}
$$

\section{Proof of the theorems}

Proof of Theorem 1.1. Using Lemma 2.2, we have

$$
p\left(\frac{q}{p-1}\right)^{\mathrm{M}}-N(p, \mathrm{M}) \leq\left|N(p, \mathrm{M})-p\left(\frac{q}{p-1}\right)^{\mathrm{M}}\right| \leq 2^{\mathrm{M}} \mathrm{M} \sqrt{p} .
$$

Thus

$$
\sqrt{p}\left(\frac{q}{p-1}\right)^{\mathrm{M}}>2^{\mathrm{M}} \mathrm{M}
$$

implies $N(p, \mathrm{M})>0$. By hypothesis, we have

$$
\frac{q}{p-1}>\frac{1}{(p-1)^{1 / 4 \mathrm{M}}}>\frac{1}{p^{1 / 4 \mathrm{M}}} .
$$

Hence (6) is satisfied if $p>2^{4 \mathrm{M}} \mathrm{M}^{4}$.

REMARK 3.1. Note that the given conditions in Theorem 1.1 ensure

$$
q>(p-1)^{1-1 / 4 \mathrm{M}} \geq\left(2^{4 \mathrm{M}} \mathrm{M}^{4}\right)^{1-1 / 4 \mathrm{M}} \geq 2^{2 \mathrm{M}^{2}} \mathrm{M}^{2} .
$$

Proof of Theorem 1.2. Write

$$
\mathrm{S}=\sum_{n \leq \mathrm{T}} \frac{1}{\phi(d)} \sum_{\chi} \bar{\chi}(a) \chi\left(n^{q}\right)
$$


where the inner sum is over all characters modulo $d$. Since

$$
\sum_{\chi} \bar{\chi}(a) \chi\left(n^{q}\right)= \begin{cases}\phi(d) & \text { if } n^{q} \equiv a(\bmod d), \\ 0 & \text { otherwise }\end{cases}
$$

S counts all solutions of (3) up to T. Further,

$$
\mathrm{S}=\sum_{n \leq \mathrm{T}} \frac{1}{\phi(d)}\left\{\sum_{\substack{\chi \\ \chi^{q}=\chi_{0}}} \bar{\chi}(a) \chi\left(n^{q}\right)+\sum_{\substack{\chi \\ \chi^{q} \neq \chi_{0}}} \bar{\chi}(a) \chi\left(n^{q}\right)\right\},
$$

where $\chi_{0}$ is the principal character modulo $d$. Thus, we have

$$
\begin{aligned}
\mathrm{S} & =\frac{n(q) \mathrm{T}}{\phi(d)}+\frac{1}{\phi(d)} \sum_{\substack{\chi \\
\chi^{q} \neq \chi_{0}}} \bar{\chi}(a) \sum_{n \leq \mathrm{T}} \chi^{q}(n) \\
& =\frac{n(q) \mathrm{T}}{\phi(d)}+O(\sqrt{d} \log d),
\end{aligned}
$$

by Pólya-Vinogradov (see page 143 of [3]). From this, we see that the main term is greater than the error term provided

$$
\mathrm{T} \gg \frac{d^{1 / 2} \phi(d) \log d}{n(q)} .
$$

Hence the theorem.

Proof of Theorem 1.4. Write

$$
\mathrm{S}=\sum_{n \leq \mathrm{T}} \frac{1}{p-1} \sum_{\chi} \bar{\chi}(a) \chi\left(f(n)^{q}\right),
$$

where the inner sum is over all characters modulo $p$. Then $\mathrm{S}$ counts the number of solutions of (4) up to T. As before, by dividing the inner sum into two parts depending on whether $\chi^{q}=\chi_{0}$ or not, we get

$$
\mathrm{S}=\frac{q \mathrm{~T}}{p-1}+\frac{1}{p-1} \sum_{\chi^{q} \neq \chi_{0}} \bar{\chi}(a) \sum_{n \leq \mathrm{T}} \chi^{q}(f(n)) .
$$

By the given hypothesis, $\left(\operatorname{order}\left(\chi^{q}\right), \operatorname{deg} f\right)=1$. Hence by Theorem 2.3 we have

$$
\mathrm{S}=\frac{q \mathrm{~T}}{p-1}+O(m \sqrt{p} \log p)
$$

This completes the proof.

Proof of Theorem 1.5. List the roots of the polynomial congruence

$$
x^{q} \equiv a(\bmod d), \quad(a, d)=1,
$$


as $r_{1}<\cdots<r_{n(q)}$. Consider the sequence $\left\{r_{i} / d\right\}$ of rational numbers in $(0,1)$. Then by the Erdős-Turán inequality (Lemma 2.5), we have

$$
|N(n(q), \alpha)-n(q) \alpha| \leq \frac{n(q)}{\mathrm{M}+1}+3 \sum_{m=1}^{\mathrm{M}} \frac{1}{m}\left|\sum_{i \leq n(q)} e\left(\frac{m r_{i}}{d}\right)\right|
$$

for any $\alpha \in(0,1)$ and $M \geq 1$. Consider the subgroup

$$
\mathrm{H}=\left\{n \in(\mathbb{Z} / d \mathbb{Z})^{*} \mid n^{q} \equiv 1(\bmod d)\right\}
$$

of $(\mathbb{Z} / d \mathbb{Z})^{*}$. Note that all roots of $(7)$ lie in a coset $b \mathrm{H}$ with $b^{q} \equiv a(\bmod d)$ of H. Hence by the theorem of Bourgain (Theorem 2.6), we have

$$
\left|\sum_{i \leq n(q)} e\left(\frac{m r_{i}}{d}\right)\right|=\left|\sum_{h \in \mathrm{H}} e\left(\frac{m b h}{d}\right)\right| \ll n(q) d^{-\varepsilon^{\prime}(\delta)} .
$$

Hence by choosing $\mathrm{M} \gg d^{\varepsilon^{\prime}(\delta)}$, we see that

$$
\begin{aligned}
\#\left\{r_{i} \mid r_{i}^{q} \equiv a(\bmod d), 0<r_{i}\right. & <\alpha d, 1 \leq i \leq n(q)\} \\
& =N(n(q), \alpha)=n(q) \alpha+O\left(n(q) d^{-\varepsilon(\delta)}\right) .
\end{aligned}
$$

Acknowledgements. It is my pleasure to thank Ram Murty for sending me his paper [9] which initiated this work and also for many valuable suggestions. I would also like to thank Purusottam Rath and the referee for their valuable comments.

\section{References}

[1] J. Bourgain, Exponential sum estimates over subgroups of $\mathbb{Z}_{q}^{*}$, q arbitrary, J. Anal. Math. 97 (2005), 317-355.

[2] D. A. Burgess, On Dirichlet characters of polynomials, Proc. London Math. Soc. (3) 13 (1963), 537-548.

[3] A. Cojocaru and M. R. Murty, An Introduction to Sieve Methods and Their Applications, Cambridge Univ. Press, Cambridge, 2006.

[4] H. Davenport, On the distribution of the lth power residues $\bmod p$, J. London Math. Soc. 7 (1932), 117-121.

[5] S. Gun, F. Luca, P. Rath, B. Sahu and R. Thangadurai, Distribution of residues modulo p, Acta Arith. 129 (2007), 325-333.

[6] M. Hudson, On the least non-residue of a polynomial, J. London Math. Soc. 41 (1966), 745-749.

[7] C. Mauduit, J. Rivat and A. Sárközy, On the pseudo-random properties of $n^{c}$, Illinois J. Math. 46 (2002), 185-197.

[8] H. Montgomery, Ten Lectures on the Interface between Analytic Number Theory and Harmonic Analysis, CBMS Reg. Conf. Ser. Math. 84, Amer. Math. Soc., 1994.

[9] M. R. Murty, Small solutions of polynomial congruences, Indian J. Pure Appl. Math. 41 (2010), 15-23.

[10] W. Schmidt, Equations over Finite Fields. An Elementary Approach, Lecture Notes in Math. 536, Springer, Berlin, 1976. 
[11] A. Weil, On the Riemann hypothesis in function fields, Proc. Nat. Acad. Sci. U.S.A. 27 (1941), 345-347.

[12] —, Sur les courbes algébriques et les variétés qui s'en déduisent, Actualités Sci. Ind. 1041, Publ. Inst. Math. Univ. Strasbourg 7 (1945), Hermann, Paris, 1948.

[13] —, On some exponential sums, Proc. Nat. Acad. Sci. U.S.A. 34 (1948), 204-207.

Sanoli Gun

Institute of Mathematical Sciences

C.I.T. campus

Taramani, Chennai 600 113, India

E-mail: sanoli@imsc.res.in

Received on 13.6.2009

and in revised form on 20.1.2010 